\title{
Boundary Value Problem for a Heated Nanofluid Flow in the Presence of Magnetic Field
}

\author{
K. HRICZÓ \\ University of Miskolc, Faculty of Mechanical Engineering and Informatics, mathk@uni-miskolc.hu
}

Abstract. The aim of this paper is to introduce some new numerical results on the magneto-thermomechanical interaction between heated viscous incompressible magnetic nanofluid and a cold wall in the presence of a spatially varying magnetic field. The governing nonlinear boundary layer equations are converted into coupled nonlinear ordinary differential equations by similarity transformation. The ODE system is solvable numerically for example using higher derivative method. The investigation is focused on the influence of governing parameters corresponding to various physical conditions. Numerical results are exhibited for the dimensionless wall skin friction and for heat transfer coefficients at the wall, along to distributions of the velocity and the temperature.

\section{Introduction}

Examination of the heat transfer in boundary layer flow is very important in our daily lives, because this phenomenon is observed in nature, where temperature difference between objects within same body exists. The boundary layer flow and energy transport phenomenon occur in viscous flows between a liquid and a solid body; moreover, it has conquered significant importance due to wide range of applications in engineering and modern industrial processes. Some of them are glass blowing, polymer extrusion, tinning and annealing of copper wires, chemical industries such as metallurgy process like metal extrusion and metal spinning, heat removal from nuclear fuel debris, artificial fibres, hot rolling and so on.

One of flowing fluids is the nanofluids, what are a stable suspension of carrier liquid and nanoparticles. These mixtures are made of various metals or non-metals e.g., aluminium (Al), copper (Cu), Silver (Ag), and graphite or carbon nanotubes respectively, and the base fluid, which includes water, oil or ethylene glycol. Ferrofluid is a special type of nanofluids, in which micron sized colloidal magnetic nanoparticles scattered and suspended consistently in a single domain non-magnetic carrier liquid. The base fluid for the ferrofluids is usually taken to be oil or water. These fluids are liquid which are highly magnetized when an external magnetic field is applied. The flow of ferromagnetic boundary layer can be controlled by changing the strengths of external magnetic field.

Several papers deal with investigating ferrofluids, as they are applicable in enhancing the heat transfer rate in several materials and liquids used in advanced technology and industry. It plays an important role in the field of chemical and electromechanical devices. Stephen [1] created and characterized the first ferromagnetic fluids in 1963 at NASA. Andersson and Valnes [2] studied heat transfer rate in ferromagnetic liquids. The effects of thermal gradients and magnetic fields are explored by Neuringer 
[3]. Albrecht et al. [4] exposed domains for the ferromagnetism and ferromagnetic effects in liquids. The free and forced convection of a magnetic fluid over a semi-infinite vertical plate, under the action of a localized magnetic field, was numerically examined by Tzirtzilakis et al. [5].

When magnetizable materials are subjected to an external magnetizing field $\mathrm{H}$, the magnetic dipoles or line currents in the material will align and create a magnetization $\mathrm{M}$.

Nowadays, the magnetohydrodynamic (MHD) flow close to infinite plate is a very popular research topic among investigators. The influence of magnetic dipole in a non-Newtonian ferrofluid was characterized for an incompressible stretchable cylinder by Awais et al. [6]. The boundary layer heat transport flow of multiphase magnetic fluid past a stretching sheet under the impact of circular magnetic field was published in [7]. Numerical investigation of magnetohydrodynamic Sisko fluid flow over linearly stretching cylinder along with combined effects of temperature depending thermal conductivity and viscous dissipation was described by Hussain et al. [8].

Neuringer [3] has studied numerically the dynamic response of ferrofluids to the application of nonuniform magnetic fields with examining the influence of magnetic field on two cases, the twodimensional stagnation point flow of a heated ferrofluid against a cold wall and the two-dimensional parallel flow of a heated ferrofluid along a wall with linearly decreasing surface temperature.

The above-mentioned results inspire this present paper, the goal is to examine the static behaviour of ferrofluids along a linearly increasing temperature wall in magnetic fields applying similarity transformation. Modelled BVP has been solved numerically in Maple by applying higher derivative method. The behaviour of physical parameters appearing in the problem are discussed with the help of figures. Changing of shear stress and heat transfer at the wall are shown by graphs.

\section{Mathematical formulation}

Consider a two-dimensional steady flow of a viscous, electrically nonconducting and incompressible nanofluid, which involves ferromagnetic (e.g. magnetite) particles over a flat surface in the horizontal direction seen in Figure 1.

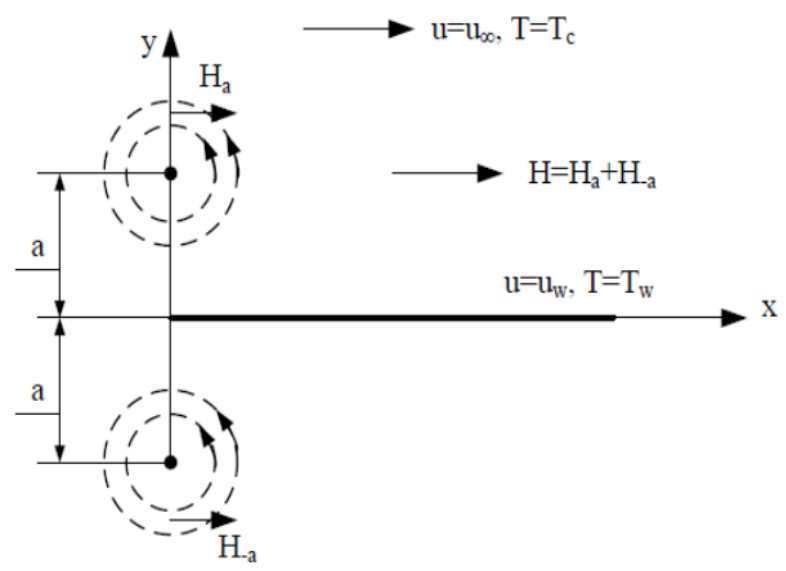

Figure 1. Parallel flow over a flat surface in magnetic field

The two magnetic dipoles are equidistant from the leading edge. The field is due to two-line currents perpendicular to and directed out of the flow plane. 
The existence of spatially varying fields is required in ferrohydrodynamic interactions [9].

The following assumptions are needed:

- the direction of magnetization of a fluid element is always in the direction of the local magnetic field,

- the fluid is electrically non-conducting and

- the displacement current is negligible.

Introducing the magnetic scalar potential $\phi$ whose negative gradient equals the applied magnetic field, i.e. $\boldsymbol{H}=-\nabla \phi$, the scalar potential can be given by

$$
\phi(x, y)=-\frac{I_{0}}{2 \pi}\left(\tan ^{-1} \frac{y+a}{x}+\tan ^{-1} \frac{y-a}{x}\right),
$$

where $I_{0}$ denotes the dipole moment per unit length and ? is the distance of the line current from the leading edge.

In the boundary layer for regions close to the wall when distances from the leading edge large compared to the distances of the line sources from the plate, i.e. $x \gg a$, then one gets

$$
[\nabla H]_{x}=-\frac{I_{0}}{\pi} \frac{1}{x^{2}}
$$

where $\mathrm{H}$ is the magnetic field.

The boundary layer equations for a two-dimensional and incompressible flow are based on expressing the conservation of mass, continuity, momentum and energy.

The analysis is based on the following four assumptions [3]:

- the applied field is of sufficient strength to saturate the ferrofluid everywhere inside the boundary layer,

- within the temperature extremes experienced by the fluid, the variation of magnetization with temperature can be approximated by a linear equation of state, the dependence of $M$ on the temperature $T$ is described by $M=K\left(T_{C}-T\right)$, where $K$ is the pyromagnetic coefficient and $T_{C}$ denotes the Curie temperature as proposed in $[3,10]$,

- the induced field resulting from the induced magnetization compared to the applied field is neglected; hence, the uncoupling of the ferrohydrodynamic equations from the electromagnetic equations and

- in the temperature range to be considered, the thermal heat capacity $c$, the thermal conductivity $k$, and the coefficient of viscosity $v$ are independent of temperature.

The governing equations are described as follows

$$
\begin{gathered}
\frac{\partial u}{\partial x}+\frac{\partial v}{\partial y}=0 \\
u \frac{\partial u}{\partial x}+v \frac{\partial u}{\partial y}=-\frac{I_{0} \mu_{0} k}{\pi \rho}\left(T_{C}-T\right) \frac{1}{x^{2}}+v \frac{\partial^{2} u}{\partial y^{2}}
\end{gathered}
$$




$$
c\left[u \frac{\partial T}{\partial x}+v \frac{\partial T}{\partial y}\right]=k \frac{\partial^{2} T}{\partial y^{2}}
$$

where $u$ and $v$ are the parallel and normal velocity components to the plate, the $x$ and $y$ axes are taken parallel and perpendicular to the plate, respectively, $v$ is the kinematic viscosity and $\rho$ denotes the density of the ambient fluid, which will be assumed constant. The system (2)-(4) of nonlinear partial differential equations is considered under the boundary conditions at the surface $(y=0)$

$$
u(x, 0)=0, v(x, 0)=0, T(x, 0)=T_{w}
$$

with increasing temperature at the wall $T_{w}=T_{C}+A x^{n+1}$ and

$$
u(x, y) \rightarrow u_{\infty}, T(x, y) \rightarrow T_{\infty}
$$

as $y$ leaves the boundary layer $(y \rightarrow \infty)$ with $T_{\infty}=T_{c}$, and $u_{\infty}$ is the exterior streaming speed which is assumed throughout the paper to be $u_{\infty}=U_{\infty} x^{n}$ (with constant $U_{\infty}$ ). Parameter $m$ is related to the power law exponent. The parameter $n=0$ refers to a linear temperature profile and constant exterior streaming speed. In case of $n=1$, the temperature profile is quadratic, and the streaming speed is linear. The value of $n=-1$ corresponds to no temperature variation on the surface.

Introducing the stream function $\psi$, defined by $u=\partial \psi / \partial y$ and $v=-\partial \psi / \partial y$, so equation (2) is automatically satisfied, and equations (3) - (4) can be formulated as

$$
\begin{aligned}
& \frac{\partial \psi}{\partial y} \frac{\partial^{2} \Psi}{\partial y x}-\frac{\partial \psi}{\partial x} \frac{\partial^{2} \psi}{\partial y^{2}}=v \frac{\partial^{3} \psi}{\partial y^{3}}-\frac{I_{0} \mu_{0} K}{\pi \rho}\left(T_{c}-T\right), \\
& C\left[\frac{\partial \psi}{\partial y} \frac{\partial T}{\partial x}-\frac{\partial \psi}{\partial x} \frac{\partial T}{\partial y}\right]=k \frac{\partial^{2} T}{\partial y^{2}} .
\end{aligned}
$$

Boundary conditions (5) and (6) are transformed to

$$
\begin{gathered}
\psi_{y}^{\prime}(x, 0)=0, \quad \psi_{x}^{\prime}(x, 0)=0, \quad T(x, 0)=T_{C}+A x^{n+1}, \\
\psi_{y}^{\prime}(x, y) \rightarrow U_{\infty} x^{n}, T(x, y)=T_{c} \text { as } y \rightarrow \infty .
\end{gathered}
$$

Now, we have two single unknown functions and two partial differential equations. The system of (8)(10) allows us to look for similarity solutions of a class of solutions $\psi$ and $T$ in the form (see [11, 12])

$$
\left.\begin{array}{c}
\psi(x, y)=B x^{b} f(\eta) \\
T=T_{C}+A x^{n+1} \Theta(\eta) \\
\eta=C x^{d} y
\end{array}\right\}
$$

where $b$ and $d$ satisfy the scaling relation $b+d=n$ and for coefficients $B$ and $C$ the relation $B / C=v$ must be fulfilled. The real numbers $b, d$ are such that $b-d=1$ and $B C=U_{\infty}$, i.e.

$$
\begin{gathered}
b=\frac{n+1}{2}, \quad d=\frac{n-1}{2}, \\
B=\sqrt{v U_{\infty}}, \quad C=\sqrt{\frac{U_{\infty}}{v} .}
\end{gathered}
$$


By considering (11), equations (7) and (8) and conditions (9) and (10) lead to the following system of coupled ordinary differential equations

$$
\begin{array}{r}
f^{\prime \prime \prime}-n f^{\prime 2}+\frac{n+1}{2} f f^{\prime}-\beta \Theta=0, \\
\Theta^{\prime \prime}+(n+1) \operatorname{Pr}\left(\frac{1}{2} f \Theta^{\prime}-\Theta f^{\prime}\right)=0
\end{array}
$$

subjected to the boundary conditions

$$
\begin{aligned}
& f(0)=0, f^{\prime}(0)=0, \Theta(0)=-1 \\
& f^{\prime}(\eta)=1, \Theta(\eta)=0 \text { as } \eta \rightarrow \infty
\end{aligned}
$$

where $\operatorname{Pr}=c v / k$ is the Prandtl number and $\beta=I_{0} \mu_{0} K A / \pi \rho U_{\infty}^{2}$ denotes the parameter of ferrohydrodynamic interaction.

The components of the non-dimensional velocity $\vec{v}=(u, v, 0)$ can be expressed by

$$
\begin{gathered}
u=U_{\infty} x^{n} f^{\prime}(\eta) \\
v=-\sqrt{v U_{\infty}} x^{\frac{n-1}{2}}\left(\frac{n+1}{2} f(\eta)+\frac{n-1}{2} f^{\prime}(\eta) \eta\right) .
\end{gathered}
$$

The physical quantities that specify the surface drag and heat transfer rate can be derived. Mathematically these quantities are interpreted in the following form

$$
\begin{gathered}
\tau_{y=0}=\rho U_{\infty} \sqrt{v U_{\infty}} x^{\frac{3 n-1}{2}} f^{\prime \prime}(0), \\
-k\left(\frac{\partial T}{\partial y}\right)_{y=0}=-k A \sqrt{\frac{U_{\infty}}{v}} x^{\frac{3 n+1}{2}} \theta^{\prime}(0),
\end{gathered}
$$

where $f^{\prime \prime}(0)$ denotes the skin friction coefficient and $\theta^{\prime}(0)$ stands for the heat transfer coefficient.

If $n=0$ and $\beta=0$, equation (12) is equivalent to the well-known Blasius equation

$$
f^{\prime \prime \prime}+\frac{1}{2} f f^{\prime}=0
$$

which appears when analyzing a laminar boundary-layer problem for Newtonian fluids [12-16].

During our investigation we suppose that the distance $x$ is greater than $a$.

Moreover, the fluid is nonelectrically conducting. The model describes the dynamics of heat transfer in an incompressible magnetic fluid under the action of an applied magnetic field. 


\section{Numerical method}

There are several computer algebraic software to solve boundary value problems of ordinary differential systems ([6], [8], [16]). One of them is the higher derivative method (HDM), which can be determined the numerical solution of boundary value problems of coupled strongly nonlinear differential equations as (12)-(13). The HDM is implemented in Maple. This method is applicable in the numerical analysis of some boundary value problems and ensure the stability by using higher derivatives [17].

The setting of digits in our case is digits $:=15$. The boundary value problem is solvable as a first order system of 7 equations, where $y 1(x)=f(\eta)$ and $y 4(x)=\Theta(\eta)$.

The left and right boundary conditions are defined by bc1 and bc2. It is necessary to give the range (bc1 to bc2) of the boundary value problem (Range:= $\left[0.0, \eta_{\max }\right]$ ). We have three parameters, $n, \beta$ and $\operatorname{Pr}$ (e.g., pars: $=\mathrm{n}=0.0, \beta=0.0, \operatorname{Pr}=10.0$ ).

The next step is to define the initial derivative in nder and the number of the nodes in nele (nder:= 3; nele := 5;). Next settings of the absolute and relative tolerance for the local error are (atol:=1e-6; rtol:= atol $/ 100 ;)$. The HDMadapt procedure is applied to determine the approximate numeric solution. The simulation gives the figure of all solution functions (from $\mathrm{y} 1$ to $\mathrm{y} 5$ ).

\section{Results and discussion}

The numerical investigation of coupled strongly nonlinear differential equations, which described a heated ferrofluid flow in magnetic field over a flat surface with boundary conditions is studied.

In order to shift the governing partial differential equations into ordinary differential equations, firstly the system needs to be simplified under usual boundary layer assumptions, after then a set of variable similarity transforms are employed. The approximate numerical solution of strongly nonlinear simultaneous equations is determined in Maple by an efficient technique with HDM method. 


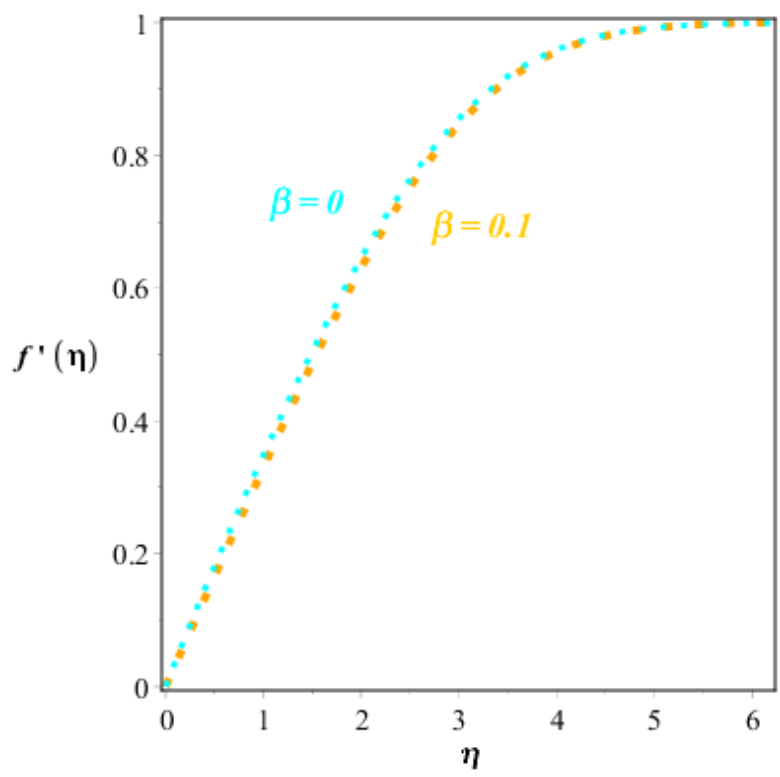

Figure 2. Velocity distribution $n=0.1, \operatorname{Pr}=10$

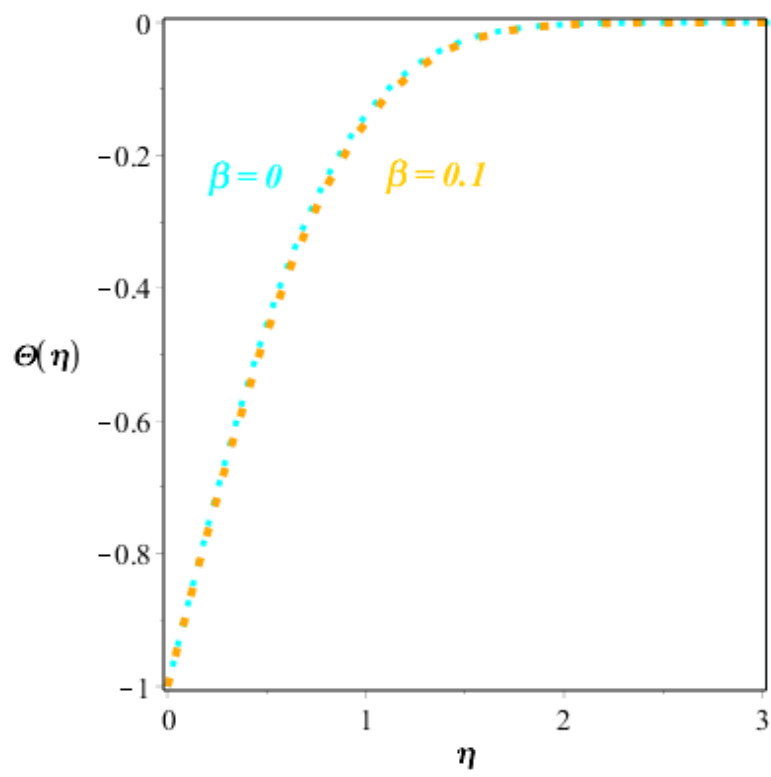

Figure 3. Temperature distribution $n=0.1, \operatorname{Pr}=10$

Numerical computations are executed, and different aspects of flow velocity and temperature are exhibited on Figs. 2-6 for different parametric conditions.

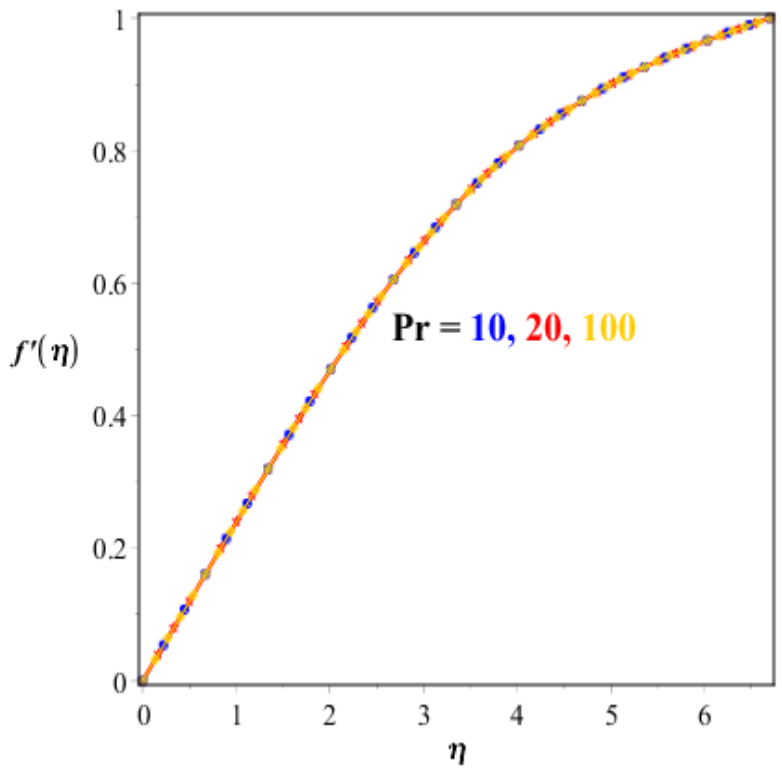

Figure 4. Velocity distribution $n=0, \beta=0.1$

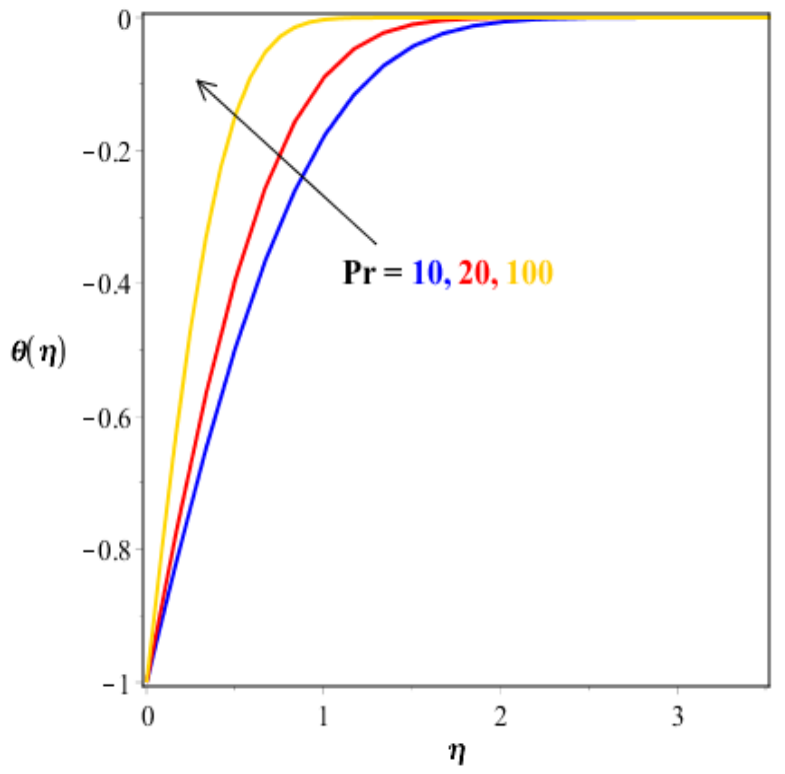

Figure 5. Temperature distribution $n=0, \beta=0.1$

Figures 2-6. show the influences of parameter $\beta$ and $\operatorname{Pr}$ for the velocity and thermal distributions in the boundary layer. If the parameter value $n=0.1$, then there is no effect of $\beta$ on the velocity and thermal distribution solutions. Significant impact on the velocity distribution of $\beta$ cannot be noticed for $n=0$. The same effect can be seen in case of Prandtl number for the velocity distribution, but there is an important effect is in case of the thermal distribution, while for increasing $\operatorname{Pr}$ the boundary layer thickness is decreasing. 
Additionally, the parameters involved in the boundary value problem influence the coefficient of skin friction and influence the flow parameters on wall shear stress. The effect of parameter $n$ is presented in Figure 6. for the shear stress at the wall, and the impact on the heat transfer at the wall in Figure 7. It can be checked from the figures that for the increasing values of $n$ the surface shear stress $f^{\prime \prime}(0)$ decreases, and the effect is same for the heat transfer rate $\theta^{\prime}(0)$ also.

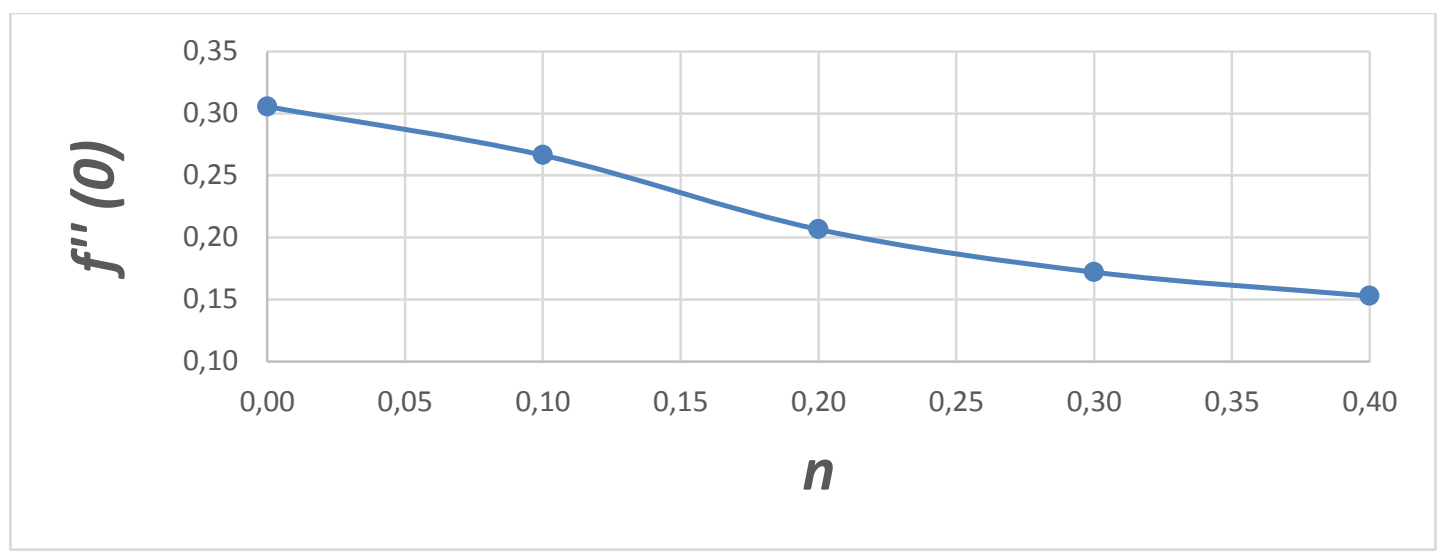

Figure 6. The shear stress at the wall $\beta=0.1, \operatorname{Pr}=10$

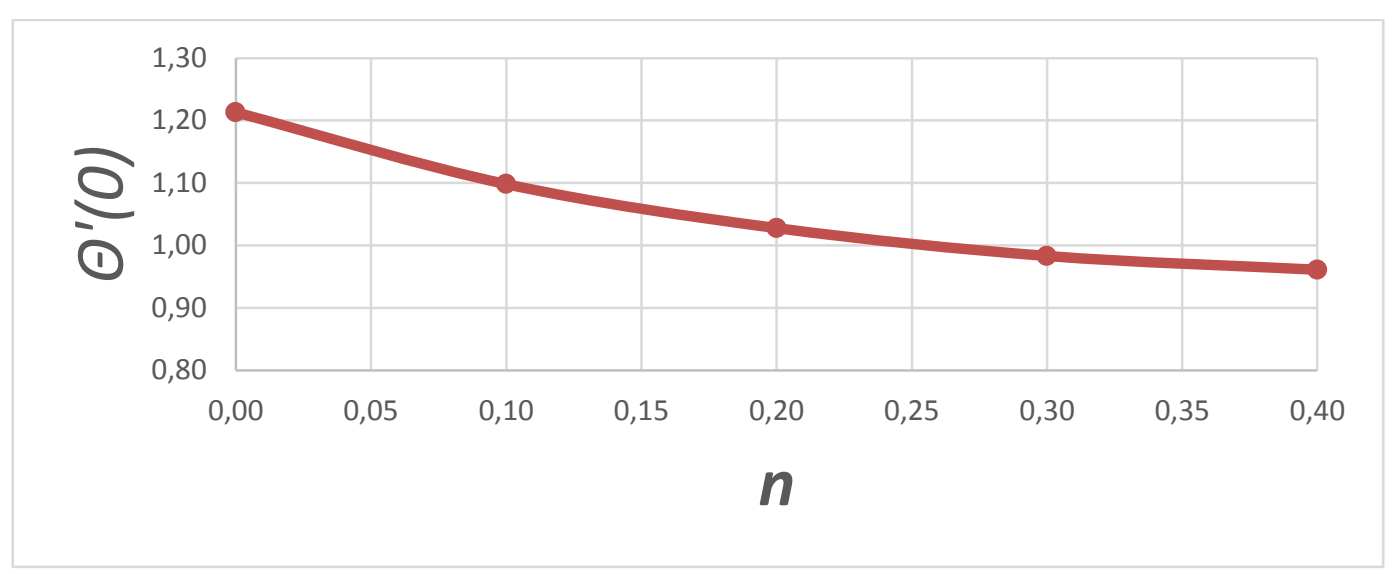

Figure 7. The heat transfer at the wall $\beta=0.1, \operatorname{Pr}=10$

Acknowledgments. Project no. 129257 has been implemented with the support provided from the National Research, Development and Innovation Fund of Hungary, financed under the $K \_18$ funding scheme.

\section{References}

[1] P. S. Stephen (1965) Low viscosity magnetic fluid obtained by the colloidal suspension of magnetic particles. US Patent 3,215,572.

[2] H. I. Andersson - O. A. Valnes (1988) Flow of a heated Ferrofluid over a stretching sheet in the presence of a magnetic dipole. Acta Mechanica. 128 pp. 39-47.

[3] J. L. Neuringer (1966) Some viscous flows of a saturated ferrofluid under the combined influence of thermal and magnetic field gradients. J. Non-linear Mech. 1 pp. 123-127. 
[4] T. Albrecht - C. Bührer - M. Fähnle - K. Maier - D. Platzek - J. Reske (1997) First observation of ferromagnetism and ferromagnetic domains in a liquid metal. Appl Phys A. 65 pp. 215-20.

[5] E.E. Tzirtzilakis - N.G. Kafoussias - A. Raptis (2010) Numerical study of forced and free convective boundary layer flow of a magnetic fluid over a flat plate under the action of a localized magnetic field. ZAMP. 61. pp. 929-947.

[6] M. Awais - M.Y. Malik - S. Bilal - T. Salahuddin - A. Hussain (2017) Magnetohydrodynamic (MHD) flow of Sisko fluid near the axisymmetric stagnation point towards a stretching cylinder. Results in Physics. 7. pp. 49-56.

[7] A. Zeeshan - A. Majeed - C. Fetecau - S. Muhammad (2017) Effects on heat transfer of multiphase magnetic fluid due to circular magnetic field over a stretching surface with heat source/sink and thermal radiation. Results in Physics. pp. 73353-3360.

[8] A. Hussain - M.Y. Malik - S. Bilal - M. Awais - T. Salahuddin (2017) Computational analysis of magnetohydrodynamic Sisko fluid flow over a stretching cylinder in the presence of viscous dissipation and temperature dependent thermal conductivity. Results in Physics. 7. pp. 139-146.

[9] J. L. Neuringer - R. E. Rosensweig (1964) Ferrohydrodynamics. Phys. Fluids. 7. pp. 1927-1937.

[10] Y. Amirat - K. Hamdache (2012) Heat Transfer in Incompressible Magnetic Fluid. J. Math. Fluid Mech. 14. pp. 217-247.

[11] G.I. Barenblatt (1996) Scaling, Self-similarity, and Intermediate Asymptotic. Cambridge Text in Applied Mathematics. Vol. 14, Cambridge University Press, Cambridge, 1996.

[12] G. Bognár (2016) On similarity solutions of MHD flow over a nonlinear stretching surface in nonNewtonian power-law fluid, Electron. J. Qual. Theory Differ. Equ. pp. 1-12.

[13] G. Bognár (2016) Magnetohydrodynamic Flow of a Power-Law Fluid over a Stretching Sheet with a Power-Law Velocity, in: Differential and Difference Equations with Applications (Springer Proceedings in Mathematics and Statistics; 164. ICDDEA, Amadora, Portugal, 2015), Springer, Bazel. pp. 131-139.

[14] G. Bognár (2012) On similarity solutions to boundary layer problems with upstream moving wall in non-Newtonian power-law fluids. IMA J. Appl. Math. 77. pp. 546-562.

[15] G. Bognár (2011) Analytic solutions to the boundary layer problem over a stretching wall. Comput. Math. Appl. 61. pp. 2256-2261.

[16] G. Bognár (2011) Analytic solutions to a boundary layer problem for non-Newtonian fluid flow driven by power-law velocity profile. WSEAS Trans. Fluid. Mech. 6. pp. 22-31.

[17] J. Chen - D. Sonawane - K. Mitra - V. R. Subramanian Yet another code for Boundary Value Problems- Higher Derivative Method, manuscript 